\title{
HISTORIA DEL ARTE DE LOS PARTOS EN EL ÁMBITO FAMILIAR
}

\author{
Manuel Jesús García Martínez
}

Dr. en Antropología Social y Cultural. Enfermero y Matrona

Prof. Unidad Docente de Matrona Virgen del Rocío (Sevilla)

Prof. E. U. Ciencias de la Salud. Universidad de Sevilla

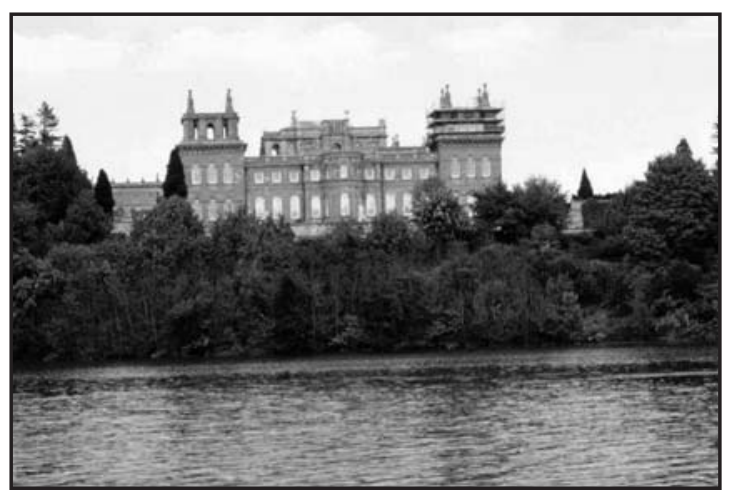

HISTORY OF ART IN CHILDBIRTH IN FAMILY ENVIRONMENT

\section{SUMMARY}

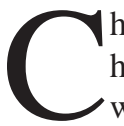

hildbirth was traditionally developed within the family environment which means where the parturient (the pregnant woman) lived her experience, personal and transferable together with her close relatives: mother, girl friends, being attended by a 'professional' - another woman or midwife well considered and recognized as such socially as professionally.

In our country, up to the latest period of XX century, midwifes were professionals in charge of attention to women in their reproductive cycle at their own homes, focussing primarily in the pregnancy, childbirth and post-partum periods. Midwifes were very much considered and well regarded women in affective and technical-scientific fields, capable of solving vital, difficult and in some cases dramatic situations with no other means than their own experience, their own skilfulness and knowledge. With new economic developments, in the sixties, in XX century and new health concepts, a fundamental change in childbearing takes place, no more faced or approached domestically, in family environments but in spe- cialized health institutions with all the implied impacts.

Important changes equally affecting the mother and her attending personnel occur, now medical doctors and specially trained midwifes would attend her. As a result the traditional ancient midwifes see their roles, functions and autonomy limited.

At present, we are facing new ways of 'thinking andregarding' childbearing with new attitudes in professionals who attend them, as well as governments who design new health policies.

Key words: Ancient midwife/ Modern midwife. Childbirth within family environment. Midwifes history. Childbearing history. Nursing history.

\section{HISTÓRIA DA ARTE DOS PARTOS NO ÂMBITO FAMILIAR}

\section{RESUMO}

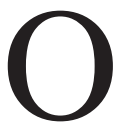
parto tem-se desenvolvido tradicionalmente no âmbito familiar, um meio no qual a parturiente tem vivido sua experiência pessoal e intransferível, em companhia de seus familiares mais próximos, mãe e amigas, sendo assistida por uma profissional, outra mulher, a parteira ou matrona, conhecida e reconhecida, tanto social quanto profissionalmente. $\mathrm{O}$ nosso país, até praticamente o último terço do passado século XX, as parteiras/matronas têm sido as profissionais encarregadas de atender à mulher durante seu ciclo reprodutiva em seu domicílio, centrando-se fundamentalmente na gravidez, parto e puerpério. Eram elas, matronas, mulheres apreciadas e valorizadas no campo afetivo e no técnico-científico, pois capazes de resolver situações vitais e comprometidas, em algum caso, dramáticas, contando apenas com sua própria experiência, perícia e conheci- 
mento. Com a chegada do desenvolvimento econômico, anos sessenta do século XX, e as novas concepções em matéria de saúde, produz-se uma mudança fundamental em relação ao fato do nascimento, realizando-se este já não no domicílio, em um ambiente familiar, senão em um centro hospitalar especializado, com todo o que este comporta. Produzem-se profundas mudanças que afetam tanto a mãe quanto o pessoal que a assistirá, matronas e médicos, tendo como conseqüência que a tradicional parteira / matrona percebe-se limitada em seu papel, suas funções e autonomia. Nestes últimos anos, estamos assistindo a novas formas de "pensar e ver" o parto, com novas atitudes por parte dos profissionais que o atendem e dos governantes que determinam as políticas sanitárias.

Palavras-chave: Parteira. Matrona; Parto no âmbito familiar; História da parteira; História dos partos; História da Enfermagem.

\section{RESUMEN}

$\mathrm{E}$ 1 parto se ha desarrollado tradicionalmente en el ámbito familiar, un medio en el que la parturienta ha vivido su experiencia, personal e intransferible, en compañía de sus familiares más cercanos, madre y amigas, siendo asistida por una profesional, otra mujer, la partera o matrona, conocida y reconocida, tanto social como profesionalmente.

En nuestro país, hasta prácticamente el último tercio del pasado siglo XX, las parteras/matronas han sido las profesionales encargadas de atender a la mujer durante su ciclo reproductivo en su domicilio, centrándose fundamentalmente en el embarazo, parto y puerperio. Eran, las matronas, mujeres apreciadas y valoradas en el campo afectivo y, en lo técnico-científico, capaces de resolver situaciones vitales comprometidas, en algún caso dramáticas, sin más ayuda que su experiencia, pericia y conocimiento.

Con la llegada del desarrollo económico, años sesenta del siglo XX, y las nuevas concepciones en materia de salud, se produce un cambio fundamental en relación al hecho del nacimiento, realizándose éste ya no en el domicilio, en un ambiente familiar, sino en un centro hospitalario especializado, con todo lo que ello conlleva. Se producen profun- dos cambios que afectan tanto a la madre como al personal que le va a asistir, matronas y médicos, teniendo como consecuencia que la tradicional partera/matrona ve recortado su papel, sus funciones y su autonomía.

En los últimos años estamos asistiendo a nuevas formas de "pensar y ver" el parto, con nuevas actitudes por parte de los profesionales que lo atienden y de los gobernantes que diseñan las políticas sanitarias.

Palabras clave: Partera. Matrona, Parto en el ámbito familiar, Historia de la matrona, Historia de los partos, Historia de la Enfermería.

\section{INTRODUCCIÓN. OBJETIVOS}

Tradicionalmente, el parto se ha desarrollado y sigue haciéndose aún en algunos países, cada vez menos- en el ámbito familiar, un medio en el que la parturienta ha vivido su experiencia, personal e intransferible, en compañía de sus familiares más cercanos, madre y amigas habitualmente, y asistida por una profesional, otra mujer, la partera o matrona, conocida y reconocida, social y profesionalmente (García Martínez, Antonio C. y otros, 1996/1997). Aunque en el campo de la cirugía y medicina los varones, denominados según la época cirujanos-comadrones o médicos obstetras, venían ejerciendo este oficio desde los siglos XVI-XVII, atendiendo los partos distócicos, es decir, aquellos que requerían una intervención quirúrgica para su desenlace, en España no fue hasta el año 1980 cuando pudo el hombre acceder legalmente a la especialidad de Asistencia Obstétrica -Matronapara Ayudantes Técnicos Sanitarios, estudios vetados hasta entonces para él (Véase Real Decreto 2287/1980).

Hasta prácticamente el último tercio del pasado siglo, las parteras/matronas han sido las profesionales encargadas de atender a la mujer durante su ciclo reproductivo en su domicilio, centrándose fundamentalmente en el embarazo, parto y puerperio. Han sido, y son, mujeres apreciadas, respetadas y valoradas por la futura madre y su familia, como la propia historia lo ha demostrado, siendo capaces, en lo técnico, gracias a su experiencia, pericia y conocimientos, de resolver situaciones vitales que podrían haber acabado con la vida de la madre, del hijo o de ambos. Sus consejos eran 
aceptados por la recién parida y su familia; sus indicaciones referentes a la dietética, lactancia, ejercicios físicos y primeros cuidados del niño se llevaban a la práctica sin discusión alguna. De ello da fe la diversa y abundante documentación de diverso tipo -religiosa, literaria, jurídica...- generada a lo largo de los siglos, así como los tratados materno-infantil que, desde el siglo XVI, se han redactado en España y en otros países.

Atendiendo al objetivo general planteado en estas VI Jornadas Internacionales de Cultura de los Cuidados, el presente trabajo pretende llevar a cabo una reflexión sobre el importante papel jugado por la familia en el hecho del nacimiento, una práctica habitual que la historia de la profesión de matrona ha puesto de manifiesto desde los orígenes del ser humano. La familia, como estructura social y unidad funcional, ha sido la responsable de prestar los cuidados necesarios durante el embarazo, parto y puerperio, siguiendo las orientaciones de la partera o matrona que llevaba a cabo el nacimiento.

\section{MATERIAL Y MÉTODO}

Se ha consultado diversa documentación depositada en tres archivos: Archivo General del Arzobispado de Sevilla, Archivo General de Indias (Sevilla) y Archivo Histórico Nacional (Madrid), así como bibliografía especializada en temas relacionados con la gestación y el nacimiento, desde un punto de vista histórico y antropológico.

En el primero de los archivos se han estudiado los Libros de Visitas Pastorales con las siguientes signaturas: 1.332-36, 1.343-46, 1.348, 1.351, $1.353,1.356$ y 1.381 , correspondientes a los años 1691 a 1715. En el Archivo Histórico Nacional se han localizado y consultado varios expedientes relacionados con el Tribunal de la Inquisición y las parteras, entre ellos uno que recoge el proceso criminal de fe contra la partera Juana Prudencia Echavarría, titulado "Relación de la causa criminal de fee que en la Inquisición de Lima se ha seguido contra Juana Prudencia Echavarría (alias) san Diego (alias) la Mama Juana, de casta samba, natural de la ciudad de la Havana, de estado viuda y de oficio cozinera, labandera y partera, por superticiosa y curandera. Año de 1779", cuya signatura es: “Inquisición de Lima”, legajo 1.649, n. 25.

En cuanto a la bibliografía utilizada, se recoge en el apartado correspondiente.
La documentación a la que hemos accedido recoge interesante información sobre la partera y su ejercicio profesional, desarrollado éste generalmente en el ámbito familiar, por lo que se constituye en una fuente de primer orden para conocer aspectos tanto del parto -forma, desarrollo...como de tradiciones, creencias y costumbres en torno al mismo.

\section{El parto en el ámbito familiar}

En el contexto de la vida cotidiana y, como consecuencia de la evolución de los lazos de sangre y el parentesco, se va a generalizar la institucionalización de la familia como estructura social esencial de convivencia y socialización primaria, responsabilizándose de los cuidados de salud de sus integrantes, del mantenimiento alimentario y de cualquier otro tipo de necesidad, incluidas las actividades socializadoras (Siles González, J., 1999). El acto del nacimiento, generalmente, necesita especialmente a la familia como agente que satisface las necesidades básicas o elementales del nuevo ser: alimentación, higiene, seguridad, etc..

Si bien la historia demuestra que el parto eutócico o normal ha transcurrido en un ámbito familiar hasta hace tan sólo unas décadas, quisiéramos hacer mención, si quiera de paso, al papel jugado por las instituciones denominadas "Casas de maternidad" o "Maternidades", surgidas a lo largo de los siglos XVIII y XIX en España y otros países europeos y que han subsistido hasta el pasado siglo XX. Como señalan Francisco Herrera y Rosa Ballester (Herrera Rodríguez, F., 1999), a estas instituciones acudían las mujeres de clases sociales más bajas a dar a luz, mujeres sin apoyo familiar, la mayoría desarraigadas socialmente, y que requerían ser atendidas al menos durante el parto y puerperio inmediato. Junto con estas Casas de maternidad, jugaron un papel no menos importante las Casas de niños expósitos, a las que acudían las madres para dejar a los recién nacidos que no podían o querían criar. En nuestro país, por hacer mención a alguna referencia legal que toca al tema que tratamos, destacamos la Ley General de Beneficencia del año 1822 -6 de febrero de 1822-, que regulaba las Juntas Provinciales y Municipales de Beneficencia y las Juntas Parroquiales, determinando el funcionamiento, distribución y clasificación de las referidas casas de maternidad, socorro, 


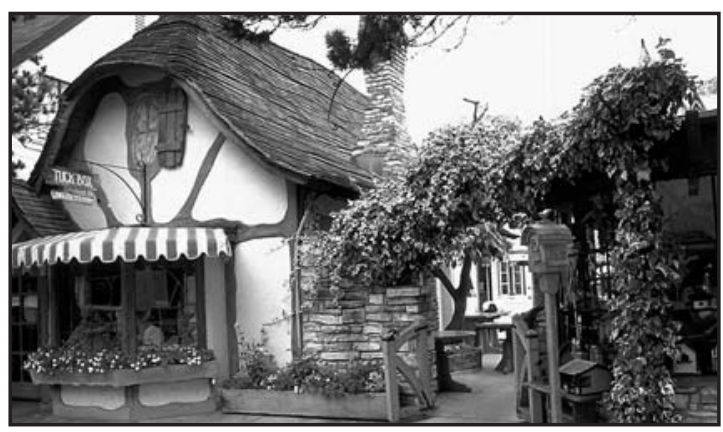

locos y hospitalidad domiciliaria, entre otros (Fernández Mérida, M. ${ }^{\mathrm{a}}$ C., 1999).

Tanto si el parto se producía en el ámbito familiar como en las citadas Casas de maternidad, el papel de la matrona ha sido fundamental en la asistencia al nacimiento de un nuevo ser, valorándose no tanto ya su formación académica, su titulación, como su buen hacer y su experiencia (García Martínez, M. J. y otros, 1994). En cuanto a lo primero, a su formación y titulación, un estudio que llevamos a cabo en el ámbito geográfico del Arzobispado de Sevilla en los siglos XVII y XVIII, utilizando como fuentes documentales los Libros de Visitas Pastorales, arrojó como resultados que de un total de 100 parteras identificadas, 79 no cumplían ningún requisito legal; 15 presentaron a las autoridades algún tipo de certificación de su formación y, finalmente, 6 asistieron el parto de manera ocasional, no teniendo este oficio como su medio de vida. Unas y otras atendían el parto en el domicilio de la futura madre, ayudadas por sus familiares, ejerciendo funciones que iban desde la extracción del feto hasta la de administrar el bautismo de urgencia "subconditione" o condicionado a aquellos niños que corrían peligro de muerte inminente.

Junto con las fuentes documentales mencionadas, los Libros de Visitas Pastorales, hemos encontrado asimismo valiosa información sobre todo lo relacionado con el desarrollo del parto en el ámbito familiar en los expedientes incoados por el Tribunal de la Inquisición a parteras, tanto en España como en América, por cometer algún delito relacionado con su oficio, siendo los más frecuentes practicar abortos, realizar actos de brujería o faltar a los preceptos de la Iglesia Católica. Uno de esos expedientes es el llevado a cabo en el año 1779 contra una partera, Juana Prudencia
Echavarría, natural de La Habana, apodada "Mama Juana", viuda y de "casta samba". Se le acusaba de supersticiosa y curandera y practicar determinados rituales hechiceriles. Se le halló culpable por el Tribunal, imponiéndosele la pena de internamiento por diez años en un hospital para mujeres, donde debía llevar a cabo su trabajo, además de realizar prácticas piadosas y espirituales, como el rezo diario del rosario y otras oraciones. Dada su avanzada edad, 65 años, se le perdonaron los azotes (García Martínez, M. J., 2008).

Con respecto a las costumbres y creencias populares asociadas al embarazo y nacimiento, el ámbito familiar se ha constituido en una fuente inagotable de información, siendo motivo de estudio de investigadores y antropólogos, como George M. Foster, quien sobre este asunto señala el alto grado de semejanza existente en la mayoría de los países hispanoamericanos. Afirma este autor que, salvo en las ciudades, es la partera o comadrona quien ayuda a la madre durante todo el período de su preñez y el nacimiento (Foster, G. M., 2002).

En nuestro país son abundantes las costumbres populares en torno a estos períodos de la vida de la mujer, quedando reflejado en las magníficas descripciones etnográficas realizadas por diversos autores, destacando entre ellas el amplio trabajo llevado a cabo por el Ateneo de Madrid en los primeros años del pasado siglo XX, recopilando más de veinte mil fichas que recogían las costumbres de nacimiento, matrimonio y muerte de la España de la época y que se conservan en el Museo Etnológico Nacional (Limón Delgado, A., 1981). Constituye este material, sin lugar a dudas, una verdadera historia del parto en el ámbito familiar, haciendo referencia a tratamientos administrados a la embarazada y parida, tradiciones religiosas en torno a la gestación y parto, primeros cuidados al recién nacido, etc. A continuación se exponen algunas de esas creencias y costumbres, recogidas de la citada Encuesta del Ateneo madrileño (Limón Delgado, A., 1981) y que hemos agrupado en los siguientes apartados: fecundación, gestación, parto y cuarentena o postparto.

\section{a) Fecundación. Remedios para ser fértil (herbo- laria, baños...)}

Existe la creencia en la localidad de Arjona (Jaén) de que la mujer que yace con varón en las 
faldas de Sierra Morena al pie del Santuario de la Virgen de la Cabeza el día del mes de mayo en que se celebra la romería de dicha Virgen, queda fecundada, y que los baños medicinales en el mar, las aguas salutíferas de Marmolejo o el coito inmediatamente después de la menstruación, son otros tantos remedios que se consideraban útiles para la fecundación.

\section{b) Gestación. Predicción del sexo}

En relación al período de gestación, destacamos la devoción a San Ramón Nonato, santo muy apreciado que vivió en el siglo XIII, apodado "non natus" (no nacido) porque su madre murió en el parto y no llegó a conocerla, siendo considerado como el patrono de las parturientas y parteras. En menor medida, las embarazadas se encomiendan a Santa Lutgarda, cuya saliva, al parecer, tenía gran poder curativo. En este capítulo destacamos también el valor de las misas y las devociones particulares como prevención del buen parto, como, por ejemplo, la costumbre en las localidades de Cazorla y Marmolejo de que la embarazada oiga una misa de pie entrada ya en el noveno mes, teniendo la creencia de que tanto como dure la misa durará el parto.

Se encuentran asimismo referencias a prescripciones de tipo físico, como pasear o la prohibición a la embarazada de realizar determinadas tareas, como la de "devanar" o "torcer" (Córdoba), labores éstas que forman parte del proceso de hilado en las cuales se usa un movimiento giratorio. Se pensaba que si no se respetaban estas indicaciones, el feto podía morir asfixiado como consecuencia de habérsele enrollado en la garganta el cordón umbilical.

No debemos olvidar en este apartado los conocidos "antojos" de las embarazadas, que se mueven entre el capricho pintoresco y las ganas de satisfacer el hambre, avalados por la creencia de que si no se llevan a efecto sufrirá las consecuencias la criatura que haya de nacer.

Por último, la predicción del sexo del niño va a ocupar un lugar importante en las costumbres populares y tradicionales, haciéndose referencia a las fases de la luna, la utilización de un pie u otro al subir una escalera, el movimiento del feto en el vientre materno o la forma que adquiere el abdomen maternal.

\section{c) Parto}

Además de encomendarse a los santos mencionados, y con el objeto de que el parto se desarrolle felizmente, hemos encontrado referencias abundantes al mundo vegetal, como la rosa de Jericó (Foster, G. M.), cuya apertura al echarla en agua es símbolo de la dilatación necesaria para dar a luz; llevar la embarazada una ristra de torvisco atada a la cintura o colocarse el "rosario de Jerusalén" sobre el vientre". Para los dolores de parto, hay costumbre de poner al cuello escapularios, medallas y amuletos de muchos tipos, y los objetos que son especialmente eficaces, señala George $\mathrm{M}$. Foster, se los prestan entre sí las madres del lugar. A los abortos se les teme y se toman diversas precauciones para su prevención, como practicar una sangría en el tobillo de la embarazada en el séptimo mes de su gestación, por pensarse que el exceso de sangre puede asfixiar al feto.

\section{d) Cuarentena/postparto}

Entre la información recogida en el estudio del Ateneo madrileño, la protección del recién nacido también ocupa un lugar importante. Se ha utilizado un gran número de objetos protectores, como lignum crucis, el torvisco, el bocado de pan en la faja, las castañas de Indias, las higas de coral y azabache, entre otros.

En el postparto, se ha venido empleando para la madre la dieta de caldo de gallina y chocolate y la misa de parida, que recuerda antiguos ritos de purificación de origen semita. No quisiéramos olvidar en este apartado el fenómeno denominado covada, término utilizado por vez primera por E. B. Tylor (1865) y que venía a señalar una curiosa costumbre caracterizada porque al nacer un niño, es el padre quien recibe los cuidados que en realidad necesita la madre, mientras ésta, sin hacer caso de su estado, continúa realizando sus ocupaciones habituales además del cuidado del recién nacido y aún del esposo, que, o bien se acuesta en una hamaca o red (pueblos primitivos de América), o en la cama (Europa), mientras los familiares y amigos vienen a felicitarle o a consolarle por los supuestos dolores que padece y que a veces exagera teatralmente, haciendo incluso una parodia del parto, dando gritos como si estuviese pariendo él mismo (Reverte Coma, José M., 1981).

La mayoría de estas costumbres, puestas en 
práctica a lo largo de los siglos, se han ido perdiendo con el paso del tiempo.

\section{Del parto en el ámbito familiar al parto hospi- talario}

Con la llegada del desarrollo económico y de las instituciones sanitarias, segunda mitad del siglo $\mathrm{XX}$, y las nuevas concepciones en materia de salud, se produce un cambio que consideramos fundamental en relación al hecho del nacimiento, realizándose éste ya no en un domicilio, en ese ambiente familiar reseñado, sino en un centro hospitalario especializado, con todo lo que ello conlleva. Estos cambios afectan tanto a la madre y a su familia como al personal que asiste el parto, médicos y matronas, los cuales disponen ahora de una excelente formación científica y emplea medios técnicos altamente sofisticados, lo cual ha permitido, sin duda alguna, disminuir el índice de mortalidad maternal e infantil, resolviéndose satisfactoriamente situaciones clínicas que hace tan sólo tres décadas era inimaginable, como recién nacidos de 500 gramos de peso o menos y de 17-18 semanas de gestación, o la resolución de determinadas patologías infecciosas, mortales por necesidad si no se tratan adecuadamente con la antibioterapia oportuna, por citar algunos ejemplos.

Consecuencia directa de ello es que la tradicional partera/matrona ve recortado su papel, sus funciones y su autonomía. De sus consejos, basados en su experiencia y la sabiduría popular a la que hemos hecho referencia brevemente, se pasa en las últimas décadas a las prescripciones e "imposiciones" de los profesionales sanitarios. El parto es considerado no ya un hecho natural o fisiológico sino un acto médico-quirúrgico, adoptándose, por ejemplo, formas de parir que podrían ser consideradas por expertos fisioterapeutas como antifisiológicas, en nada parecidas a las que tradicionalmente se han venido realizando. De colaborar activamente la parturienta en el desarrollo del parto se ha pasado a una actitud más pasiva o totalmente pasiva en algunos casos. En no pocas ocasiones, la intimidad de la mujer se ve sustituida por una situación de intimidación desarrollada en un espacio frío, a veces deshumanizado, lejos de ese ambiente familiar del que proviene la recién parida.

No obstante lo dicho, puede apreciarse en los últimos años nuevas formas de "pensar y ver" el parto, con nuevas actitudes por parte de los profesionales que lo atienden y de los gobernantes que diseñan las políticas sanitarias, conscientes de la importancia de recuperar el "parto natural". Hemos de citar aquí inexcusablemente la labor de determinadas asociaciones y la iniciativa de profesionales, matronas y personal médico, por volver a realizar el parto en ese ambiente familiar de décadas anteriores. Así, encontramos una preocupación creciente por abordar temas como el de la humanización del parto, el papel más activo de la mujer que pare y de sus seres queridos, verdaderos protagonistas del mismo, no lo olvidemos, aplicándose técnicas de relajación y respiración y otras formas de parir, caso del parto en el agua, del que ya se hablaba a principios del siglo XIX, con menos intervención instrumental y medicación, rescatándose el carácter íntimo y familiar, aunque sea en el propio hospital, aunando la sabiduría atesorada de las matronas durante siglos con el desarrollo científico y técnico del momento presente.

\section{CONCLUSIONES}

Como síntesis de lo expuesto y, a modo de conclusiones, podemos decir que ha sido, por tanto, el ámbito familiar donde ha transcurrido históricamente el parto, y que han sido la partera/matrona junto con la familia los responsables de prestar los cuidados necesarios durante los períodos del embarazo, parto y puerperio.

Uno de los objetivos específicos de estas VI Jornadas es el de "Identificar los aspectos esenciales de las funciones cuidadoras de la familia a través de la historia". En este sentido, el hecho de parir en casa, según nos enseña la Historia, ha supuesto para la parturienta: un mayor grado de autonomía de movimiento y poder de decisión; tranquilidad por conocer y dominar el espacio físico en que se mueve, disminuyendo notablemente así la ansiedad que inevitablemente genera el parto; la ayuda directa de la familia, jugando ésta un papel central en las distintas vertientes del cuidado, sobre todo en el apoyo psicológico; un mayor grado de intimidad, celosamente custodiada por otra mujer, la matrona, y por las otras mujeres de la familia; desarrollo de un parto más natural, adoptando posturas más cómodas y fisiológicas que las que se hacen en un centro hospitalario, bien en la silla de parir, bien en su propia cama $y$, finalmente, la seguridad que 
transmite el hecho de ser atendida durante su embarazo, parto y postparto por una profesional que acumula arte y experiencia.

Compartimos plenamente el Objetivo General de estas Jornadas en el sentido de que se hace necesario contar con las aportaciones desde la Historia, la Antropología y demás Ciencias Sociales, para no sólo comprender la evolución, el desarrollo del parto, las formas de parir y las creencias y costumbres que rodean esos momentos trascendentales de la vida del ser humano, sino, además, enriquecerlo, aplicando los distintos enfoques y paradigmas relacionados con la función cuidadora de la familia, sin olvidar la aportación de los profesionales que, como la partera o matrona, han hecho en beneficio de las mujeres. Con ello, aprovecharíamos esa sabiduría, el "capital humano", referido por Schultz, o el "capital social", de Bourdieu, atesorado durante tantos siglos. La puesta en valor de todo este patrimonio o capital repercutirá, estamos seguro de ello, en beneficio de un mejor parto y una experiencia inolvidable para la madre y su familia.

\section{NOTAS Y BIBLIOGRAFÍA}

El presente trabajo fue expuesto en la Mesa Redonda titulada "Historia de la Enfermería y cuidados familiar y comunitario", dentro del marco de las VI Jornadas Internacionales de Cultura de los Cuidados. IX Reunión de Investigación Cualitativa, organizadas por la Asociación Nacional de Historia y Antropología de los Cuidados y el Ilustre Colegio Oficial de Enfermería de Alicante. Alicante, 12 y 13 de junio de 2008.

- Cabré, Montserrat; Ortiz, Teresa (eds.) (2001), Sanadoras, matronas y médicas en Europa. Siglos XII-XX. Icaria Editorial, S. A. Barcelona.

- Fernández Mérida, M. ${ }^{a}$ C. (1998/1999) "La asistencia domiciliaria y la legislación del siglo XIX”. En Híades. Revista de historia de la Enfermería, n. ${ }^{\circ}$ 5-6. Alcalá de Guadaíra (Sevilla), pp. 145-154.

- Foster, George M. (2002), La cultura tradicional en España y América. Signatura Ediciones de Andalucía, S. L. Sevilla, p. 211.

- Foucault, Michel (1999), El nacimiento de la clínica. Una arqueología de la mirada médica. Siglo Veintiuno de España Editores. Madrid.

- García Martínez, M. J. (2008), "Documentos para la historia de las matronas en América: El proceso de fe contra Juana Prudencia Echavarría. Lima, 1778". En Híades. Revista de historia de la Enfermería, n. ${ }^{\circ} 10$. Alcalá de Guadaíra (Sevilla), pp. 1007-1044.
- García Martínez, Manuel J. y otros (1994), "Registro y control de las matronas por la Iglesia Hispalense. (La imagen de la matrona a través de los Libros de Visitas Pastorales del Arzobispado de Sevilla, siglos XVII y XVIII)". En Híades. Revista de historia de la Enfermería, n. ${ }^{\circ}$ 1. Alcalá de Guadaíra (Sevilla), pp. 13-33.

- García Martínez, Antonio C. y otros (1996/1997), "La imagen de la matrona en la Baja Edad Media”. En Híades. Revista de historia de la Enfermería, n. ${ }^{\circ}$ 3-4. Alcalá de Guadaíra (Sevilla), pp. 61-82.

- García Martínez, Manuel J.; García Martínez, Antonio C. (1998/1999), "Fechas claves para la historia de las Matronas en España". En Híades. Revista de historia de la Enfermería, n. ${ }^{\circ}$ 5-6. Alcalá de Guadaíra (Sevilla), pp. 243-260.

- Herrera Rodríguez, F. (1999), "La Casa de Maternidad de Cádiz a finales del siglo XIX". En Híades. Revista de historia de la Enfermería, n. ${ }^{\circ}$ 5-6. Alcalá de Guadaíra (Sevilla), pp. 271-284.

- Limón Delgado, A. (1981), Costumbres populares andaluzas de nacimiento, matrimonio y muerte. Servicio de Publicaciones de la Excma. Diputación Provincial de Sevilla. Sevilla, 1981. El cuestionario original, desaparecido durante la Guerra Civil Española, llevaba por título: "Información promovida por la Sección de Ciencias Morales y Políticas del Ateneo de Madrid, en el campo de las costumbres populares y en los tres hechos más característicos de la vida: el nacimiento, el matrimonio y la muerte". Tres años después de recibidas las respuestas de los corresponsales, en 1905, ve la luz el primer y mejor trabajo que se ha hecho aprovechando esta magnífica información: el libro de Rafael Salillas titulado La Fascinación en España, con el subtítulo "Brujas, brujería y amuletos". Véase Limón Delgado, p. 10.

- Reverte Coma, José M. (1981), Antropología Médica I. Editorial Rueda. Madrid, p. 403.

- Sánchez Bayle, Marciano (ed.) (1996), El sistema sanitario en España. Evolución, situación actual, problemas y perspectivas. Ed. Los Libros de la Catarata. Madrid.

- Siles González, José (1999), Historia de la Enfermería. Editorial Aguaclara. Alicante, p. 109.

\section{FUENTES}

- Libros de Visitas Pastorales núms. 1.332-36, 1.343-46, 1.348, $1.351,1.353,1.356$ y 1.381 , correspondientes a los años 1691 a 1715. Archivo General del Arzobispado de Sevilla.

- "Relación de la causa criminal de fee que en la Inquisición de Lima se ha seguido contra Juana Prudencia Echavarría (alias) san Diego (alias) la Mama Juana, de casta samba, natural de la ciudad de la Havana, de estado viuda y de oficio cozinera, labandera y partera, por superticiosa y curandera. Año de 1779”. Signatura: “Inquisición de Lima”, legajo 1.649, n. . 25. Archivo Histórico Nacional.

- Real Decreto 2287/1980, de 26 de septiembre (BOE núm. 258, de 27 de octubre de 1980). En su Artículo primero, recoge: "Los Ayudantes Técnicos Sanitarios, tanto masculinos como femeninos, podrán cursar la especialidad de Asistencia Obstétrica establecida por el Decreto de dieciocho de enero de mil novecientos cincuenta y siete, y obtener el diploma correspondiente, quedando suprimida la limitación por razón del sexo en él contenida". Se suprime, también, la obligatoriedad de seguir las enseñanzas en régimen de internado. 


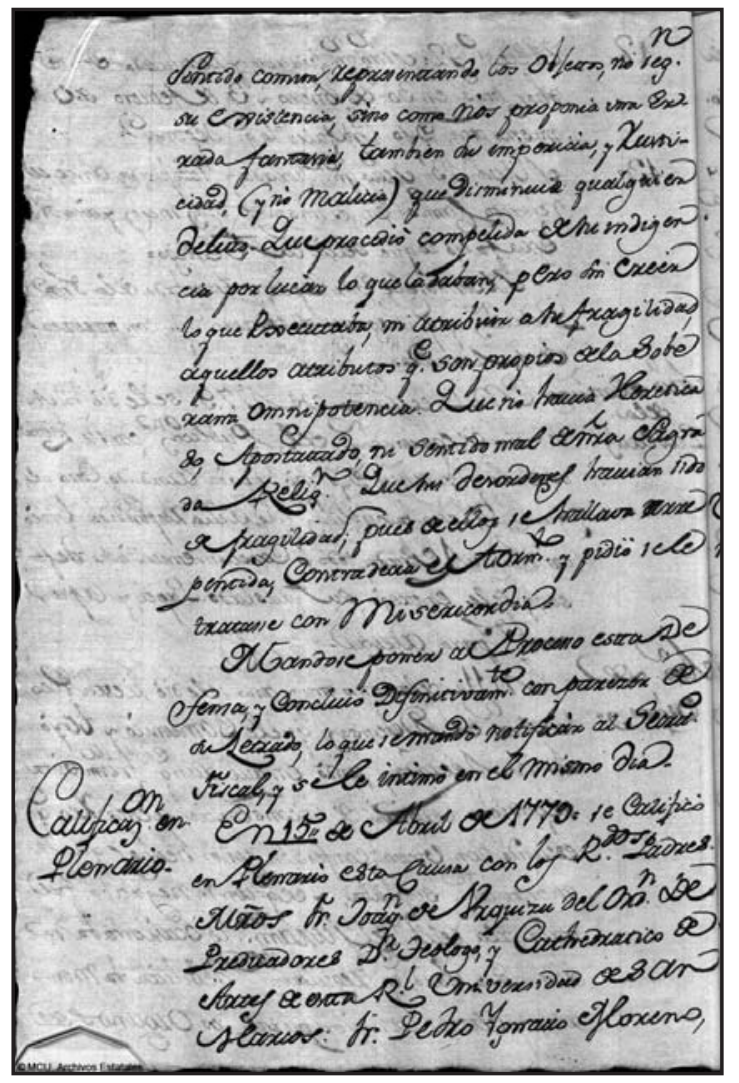

Proceso criminal seguido contra la partera Juana Prudencia Echavarría en Lima en el año 1779. Archivo Histórico Nacional (Madrid). “Inquisición de Lima”. Legajo 3, n. ${ }^{o}$ 25, f. 12v.

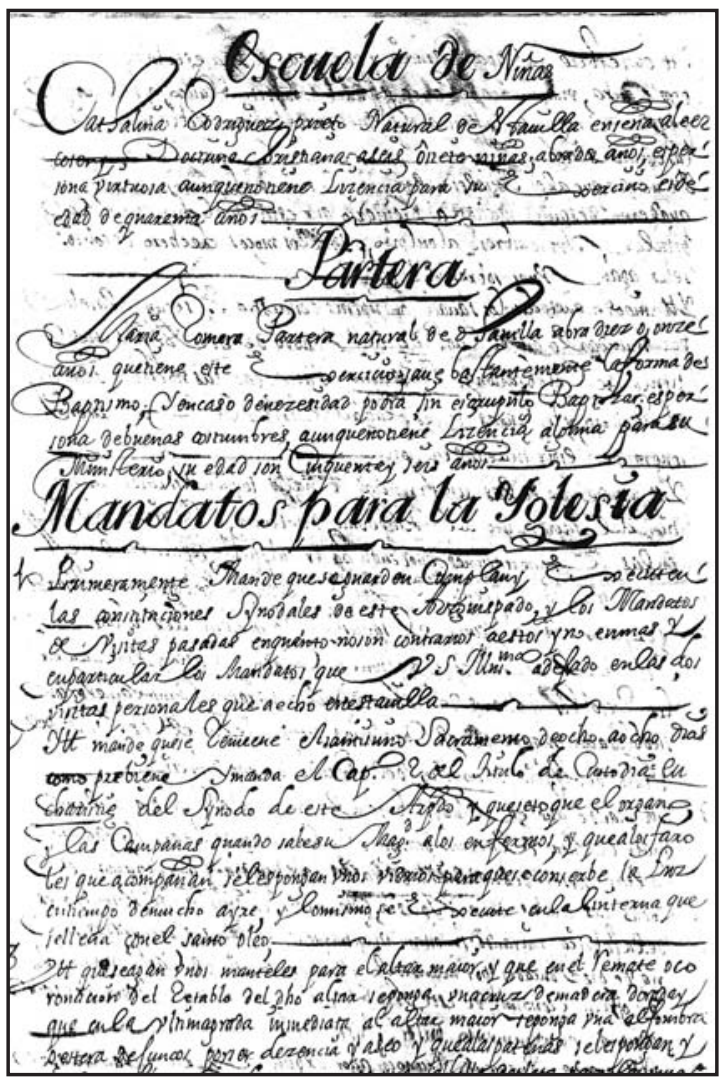

Libro de Visitas Pastorales del Archivo General del Arzobispado de Sevilla (siglo XVII). En ellos queda reflejado funciones de la partera tradicional, tales como la administración del bautismo de urgencia a los recién nacidos en peligro de muerte.

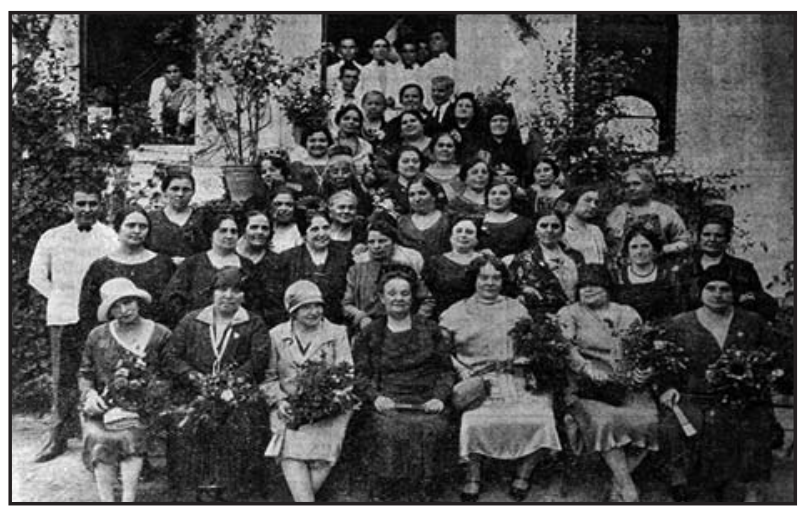

Las matronas han estado atendiendo los partos en el ámbito familiar hasta prácticamente el último tercio del siglo pasado. Fotografía realizada el 29-IX-1929 en Sevilla, en la que se muestran matronas representantes de diversos Colegios provinciales de matronas de España. 